\title{
Dynamical properties of weighted translation operators on the Schwartz space $\mathcal{S}(\mathbb{R})$
}

\author{
Michał Goliński ${ }^{1}$ D Adam Przestacki ${ }^{1}$
}

Received: 17 July 2018 / Accepted: 18 March 2019 / Published online: 1 April 2019

(C) The Author(s) 2019

\begin{abstract}
In this paper we investigate the dynamical properties of weighted translation operators acting on the Schwartz space $\mathcal{S}(\mathbb{R})$ of rapidly decreasing functions, i.e., operators of the form $T_{w}: \mathcal{S}(\mathbb{R}) \rightarrow \mathcal{S}(\mathbb{R}), f(\cdot) \mapsto w(\cdot) f(\cdot+1)$. We characterize when those operators are hypercyclic, weakly mixing, mixing and chaotic. Several examples illustrate our results and show which of those classes are different.
\end{abstract}

Keywords Linear dynamics $\cdot$ Schwartz space $\cdot$ Hypercyclicity $\cdot$ Weighted translation operator

Mathematics Subject Classification 47B33 · 47A16 · 46E10

\section{Introduction}

One of the most important spaces of classical analysis is the Schwartz space $\mathcal{S}(\mathbb{R})$ of rapidly decreasing functions, i.e., the space consisting of all smooth functions $f: \mathbb{R} \rightarrow \mathbb{C}$ such that $\|f\|_{N}<\infty$ for every $N \in \mathbb{N}$, where $\|\cdot\|_{N}$ is the norm on $\mathcal{S}(\mathbb{R})$ defined by the formula

$$
\|f\|_{N}=\max _{0 \leq i \leq N} \sup _{x \in \mathbb{R}}\left(1+x^{2}\right)^{N}\left|f^{(i)}(x)\right| .
$$

The authors research was partially supported by National Science Centre (Poland) Grant UMO-2013/10/A/ST1/00091.

$\bowtie \quad$ Michał Goliński

golinski@amu.edu.pl

Adam Przestacki

adamp@amu.edu.pl

1 Faculty of Mathematics and Computer Science, Adam Mickiewicz University, ul. Umultowska 87, 61-614 Poznan, Poland 
It is well known that the space $\mathcal{S}(\mathbb{R})$ equipped with the locally convex topology generated by the family of norms $\left\{\|\cdot\|_{N}: N \in \mathbb{N}\right\}$ is a Fréchet space. The goal of this paper is to investigate the dynamical properties of a natural class of operators acting on this space, namely the class of weighted translation operators

$$
T_{w}: \mathcal{S}(\mathbb{R}) \rightarrow \mathcal{S}(\mathbb{R}), f(\cdot) \mapsto w(\cdot) f(\cdot+1),
$$

where $w: \mathbb{R} \rightarrow \mathbb{C}$ is a smooth function. It is easy to see that such an operator is well defined (and thus continuous by the Closed Graph Theorem) if and only if the function $w$ belongs to the space of smooth functions of moderate growth, i.e., the space $\mathcal{O}_{M}(\mathbb{R})$ consisting of all smooth functions $f: \mathbb{R} \rightarrow \mathbb{C}$ such that for every $k \geq 0$ there is $l \in \mathbb{N}$ with $\left|w^{(k)}(x)\right|<l\left(1+x^{2}\right)^{l}$ for every $x \in \mathbb{R}$. The space $\mathcal{O}_{M}(\mathbb{R})$ is also known as the space of multipliers of $\mathcal{S}(\mathbb{R})$ since $w \in \mathcal{O}_{M}(\mathbb{R})$ if and only if for every $f \in \mathcal{S}(\mathbb{R})$ also $w \cdot f \in \mathcal{S}(\mathbb{R})$.

Let us briefly recall the basic concepts of linear dynamics. Let $X$ be a Fréchet space and let $T \in L(X)$ be a continuous linear operator. For every $n \geq 1$ the operator $T^{n}: X \rightarrow X$ is defined as the $n$th iterate of $T$, i.e,

$$
T^{n}:=\underbrace{T \circ \cdots \circ T}_{n \text { times }} .
$$

The operator $T: X \rightarrow X$ is called hypercyclic if there exists $x \in X$ such that the set

$$
\operatorname{orb}(x, T):=\left\{T^{n} x: n \geq 1\right\}
$$

is dense in $X$. Such an element $x$ is called a hypercyclic vector of $T$. By the famous Birkhoff's Transitivity Theorem, an operator $T$ acting on a separable Fréchet space $X$ is hypercyclic if and only if it is topologically transitive, i.e., for every two nonempty open sets $U, V \subset X$ there is $n \in \mathbb{N}$ such that $T^{n}(U) \cap V \neq \emptyset$. The operator $T$ is called weakly mixing if the operator $T \times T$ is topologically transitive, i.e., for every four nonempty open sets $U_{1}, U_{2}, V_{1}, V_{2} \subset X$ there is $n \in \mathbb{N}$ such that $T^{n}\left(U_{1}\right) \cap V_{1} \neq$ $\emptyset$ and $T^{n}\left(U_{2}\right) \cap V_{2} \neq \emptyset$. The operator $T$ is called mixing if for every two nonempty open sets $U, V \subset X$ there is $N \in \mathbb{N}$ such that $T^{n}(U) \cap V \neq \varnothing$ for every $n \geq N$. Finally, $T$ is called chaotic if it is hypercyclic and has a dense set of periodic points (a point $x \in X$ is called a periodic point of $T$ if $T^{k} x=x$ for some $k \in \mathbb{N}$ ). From the very definitions it is clear that every mixing operator is weakly mixing and every weakly mixing operator is hypercyclic. For a detailed exposition of the subject of linear dynamics we refer to the monographs $[1,9]$.

The first example of a hypercyclic operator goes back to Birkhoff who proved that the translation operator $T: H(\mathbb{C}) \rightarrow H(\mathbb{C}), f(\cdot) \mapsto f(\cdot+1)$ is hypercyclic (see [2]). In fact Birkhoff's operator is an example of a composition operator and the dynamics of this kind of operators was later on studied by various authors (see [8] for composition operators on spaces of holomorphic functions, see [3] for composition operators on spaces of real analytic functions, see [10] for weighted composition operators on Banach spaces of continuous functions and $L_{p}$ spaces, see [11] for weighted composition operators on the space of smooth functions). Surprisingly, as shown below, 
composition operators are never hypercyclic when acting on the space $\mathcal{S}(\mathbb{R})$. Let us note that recently (see [6]) a description was found of those smooth functions $\psi: \mathbb{R} \rightarrow \mathbb{R}$ for which the composition operator $C_{\psi}: \mathcal{S}(\mathbb{R}) \rightarrow \mathcal{S}(\mathbb{R}), f \mapsto f \circ \psi$ is well defined.

Fact 1 Let $\psi: \mathbb{R} \rightarrow \mathbb{R}$ be a smooth function such that the composition operator $C_{\psi}: \mathcal{S}(\mathbb{R}) \rightarrow \mathcal{S}(\mathbb{R}), f \mapsto f \circ \psi$ is well defined. Then $C_{\psi}$ is not hypercyclic.

Proof Let $f \in \mathcal{S}(\mathbb{R})$ be arbitrary. There exists $M>0$ such that $|f(x)|<M$ for every $x \in \mathbb{R}$. If $n \in \mathbb{N}$, then $\left|\left(C_{\psi}^{n} f\right)(x)\right|<M$ for every $x \in \mathbb{R}$. This clearly implies that $C_{\psi}$ is not hypercyclic.

The second important example of a hypercyclic operator goes back to MacLane who proved that the differential operator $D: H(\mathbb{C}) \rightarrow H(\mathbb{C}), f \mapsto f^{\prime}$ is hypercyclic (this was later on extended even to infinite order differential operators on $H(\mathbb{C})$, see [4]). By easy arguments from linear dynamics, differential operators are also hypercyclic on the space of smooth functions on the real line. This is not the case if we consider these operators on $\mathcal{S}(\mathbb{R})$.

Fact 2 Let $a_{0}, \ldots, a_{n}$ be a sequence of complex numbers and let

$$
P: \mathcal{S}(\mathbb{R}) \rightarrow \mathcal{S}(\mathbb{R}), P(f)=\sum_{i=0}^{n} a_{i} f^{(i)}
$$

Then $P$ is not hypercyclic.

Proof Let $\phi: \mathcal{S}(\mathbb{R}) \rightarrow \mathbb{C}$ be the continuous linear functional defined on $\mathcal{S}(\mathbb{R})$ by the formula

$$
\phi(f)=\int_{-\infty}^{\infty} f(x) d x .
$$

Then for every $n \in \mathbb{N}$

$$
\phi\left(P^{n} f\right)=a_{0}^{n} \int_{-\infty}^{\infty} f(x) d x
$$

If $f$ would be a hypercyclic vector for $P$, then the set $\left\{\phi\left(P^{n} f\right): n \in \mathbb{N}\right\}$ should be dense in $\mathbb{C}$. This is impossible.

The above considerations show that many classical hypercyclic operators are not hypercyclic when considered on the Schwartz space. Our aim was to find a natural class of operators acting on $\mathcal{S}(\mathbb{R})$ and to study them from the point of view of linear dynamics. Motivated by weighted bilateral shifts acting on sequence spaces, we decided to study weighted translation operators on $\mathcal{S}(\mathbb{R})$. In Theorem 1 we give a characterization of hypercyclic weighted translation operators. It is not a surprise that this class is equal to the class of weakly mixing weighted translation operators. In Theorems 2 and 3 we 
characterize the class of mixing and chaotic weighted translation operators. In fact, in Theorem 4 we show that those classes are equal. In the last sections we present some tools which help to decide if a given function $w$ induces an operator $T_{w}$ with some dynamical properties. Using those tools we construct examples showing which of the considered classes of weighted translation operators are different.

\section{Hypercyclic, weakly mixing, mixing and chaotic weighted translation operators}

In this section we characterize the dynamical properties of weighted translation operators acting on the $S c h w a r t z$ space $\mathcal{S}(\mathbb{R})$. In the first theorem we show that hypercyclicity of these operators is equivalent to weak mixing. This is not surprising since for many natural operators the situation is the same (see [11] for weighted composition operators on the space of smooth functions, see [10] for weighted composition operators on Banach spaces of continuous and integrable functions, see [12] and [7] for weighted backward shifts and weighted bilateral shifts on sequence spaces). Let us emphasize that there are hypercyclic operators which are not weakly mixing (see [5]).

Theorem 1 Let $w \in \mathcal{O}_{M}(\mathbb{R})$. The following conditions are equivalent:

(i) The operator $T_{w}: \mathcal{S}(\mathbb{R}) \rightarrow \mathcal{S}(\mathbb{R}), f(\cdot) \mapsto w(\cdot) f(\cdot+1)$ is weakly mixing.

(ii) The operator $T_{w}: \mathcal{S}(\mathbb{R}) \rightarrow \mathcal{S}(\mathbb{R}), f(\cdot) \mapsto w(\cdot) f(\cdot+1)$ is hypercyclic.

(iii) The following conditions are satisfied:

(a) For every $x \in \mathbb{R}$ we have $w(x) \neq 0$.

(b) For every compact set $K \subset \mathbb{R}$ there exists an increasing sequence of natural numbers $\left(n_{k}\right)_{k \in \mathbb{N}}$ such that for every $j, l \geq 0$

$$
\sup _{x \in K-n_{k}}\left(1+x^{2}\right)^{j}\left|\left(\prod_{n=0}^{n_{k}-1} w(x+n)\right)^{(l)}\right| \stackrel{k \rightarrow \infty}{\longrightarrow} 0
$$

and

$$
\sup _{x \in K+n_{k}}\left(1+x^{2}\right)^{j}\left|\left(\frac{1}{\prod_{n=1}^{n_{k}} w(x-n)}\right)^{(l)}\right| \stackrel{k \rightarrow \infty}{\longrightarrow} 0 .
$$

Proof $(i) \Rightarrow$ (ii) This is obvious since every weakly mixing operator is hypercyclic. (ii) $\Rightarrow$ ( iii) To prove that (a) holds, let us assume to the contrary that there is $x_{0} \in \mathbb{R}$ such that $w\left(x_{0}\right)=0$. Then for every $n \in \mathbb{N}$ and $f \in \mathcal{S}(\mathbb{R})$ we have $T_{w}^{n}(f)\left(x_{0}\right)=0$. This shows that $T_{w}$ cannot have a dense orbit.

In order to prove that hypercyclicity of $T_{w}$ implies condition $(b)$, let us fix a compact set $K \subset \mathbb{R}$. Without loss of generality we may assume that $K=[-D, D]$ for some $D>0$. It is easy to see that is enough to show that for every $\varepsilon>0$ and every $N \in \mathbb{N}$ there is a natural number $k \geq N$ such that for every $0 \leq l \leq N$ 


$$
\sup _{x \in K-k}\left(1+x^{2}\right)^{N}\left|\left(\prod_{n=0}^{k-1} w(x+n)\right)^{(l)}\right|<\varepsilon
$$

and

$$
\sup _{x \in K+k}\left(1+x^{2}\right)^{N}\left|\left(\frac{1}{\prod_{n=1}^{k} w(x-n)}\right)^{(l)}\right|<\varepsilon .
$$

To prove the desired statement let us consider the set $U \subset \mathcal{S}(\mathbb{R})$ consisting of all functions $f$ such that

$$
1<\left|f^{(l)}(x)\right|<M \text { for } x \in[-D, D], \quad 0 \leq l \leq N
$$

and

$$
\max _{0 \leq l \leq N} \sup _{x \in \mathbb{R} \backslash[-D-1, D+1]}\left(1+x^{2}\right)^{N}\left|f^{(l)}(x)\right|<C \varepsilon,
$$

where

$$
M=2 \max _{0 \leq i \leq N} \frac{(N+1) !(2 D+2)^{N+1-i}}{(N+1-i) !}
$$

and $C$ is a positive constant depending on $D, N$ and $w$ that will be fixed later.

Claim 1 The set $U$ is nonempty.

Proof of the claim. Any smooth function supported on $[-D-1, D+1]$ which is equal to the polynomial

$$
(x+D+2)^{N+1}
$$

on the interval $[-D, D]$ belongs to $U$.

Claim 2 The set $U$ is open.

Proof of the claim. Let $g \in U$. Then there exists $\delta>0$ such that

$$
1+\delta<\left|g^{(l)}(x)\right|<M-\delta \text { for } x \in[-D, D], \quad 0 \leq l \leq N
$$

and

$$
\max _{0 \leq l \leq N} \sup _{x \in \mathbb{R} \backslash[-D-1, D+1]}\left(1+x^{2}\right)^{N}\left|g^{(l)}(x)\right|<C \varepsilon-\delta .
$$

One can easily check that

$$
\left\{f \in \mathcal{S}(\mathbb{R}):\|f-g\|_{N}<\delta\right\} \subset U
$$

which proves the claim. 
Claim 3 There is $k \geq N$ such that the inequalities (1) and (2) hold for $0 \leq l \leq N$. Proof of the claim: By Claim 1 and Claim 2 the set $U$ is nonempty and open. Since $T_{w}$ is hypercyclic, we can find $k \in \mathbb{N}$ large enough to ensure that

$$
T_{w}^{k}(U) \cap U \neq \emptyset \text { and }[-D, D] \pm k \cap[-D+1, D+1]=\emptyset .
$$

Let $f \in U$ be such that $T_{w}^{k}(f) \in U$. By (4), we obtain that for $0 \leq l \leq N$

$$
\begin{aligned}
& \sup _{x \in K-k}\left(1+x^{2}\right)^{N}\left|\left(T_{w}^{k}(f)\right)^{(l)}(x)\right| \\
& \leq \sup _{x \in \mathbb{R} \backslash[-D-1, D+1]}\left(1+x^{2}\right)^{N}\left|\left(T_{w}^{k}(f)\right)^{(l)}(x)\right|<C \varepsilon .
\end{aligned}
$$

Using the product rule we obtain that for $0 \leq l \leq N$

$$
\sup _{x \in K-k}\left(1+x^{2}\right)^{N}\left|\sum_{i=0}^{l}\left(\begin{array}{l}
l \\
i
\end{array}\right)\left(\prod_{n=0}^{k-1} w(x+n)\right)^{(l-i)} f^{(i)}(x+k)\right|<C \varepsilon .
$$

For $l=0$ this gives

$$
\sup _{x \in K-k}\left(1+x^{2}\right)^{N}\left|\left(\prod_{n=0}^{k-1} w(x+n)\right) f(x+k)\right|<C \varepsilon
$$

and for $1 \leq l \leq N$

$$
\begin{aligned}
& \sup _{x \in K-k}\left(1+x^{2}\right)^{N}\left|\left(\prod_{n=0}^{k-1} w(x+n)\right)^{(l)} f(x+k)\right| \\
& \quad<C \varepsilon+\sum_{i=1}^{l}\left(\begin{array}{l}
l \\
i
\end{array}\right) \sup _{x \in K-k}\left(1+x^{2}\right)^{N}\left|\left(\prod_{n=0}^{k-1} w(x+n)\right)^{(l-i)} f^{(i)}(x+k)\right| .
\end{aligned}
$$

Since $f \in U$, it satisfies the condition in (3), and we obtain from (5) that

$$
\sup _{x \in K-k}\left(1+x^{2}\right)^{N}\left|\prod_{n=0}^{k-1} w(x+n)\right|<C \varepsilon
$$


and from (6), for $1 \leq l \leq N$, we get

$$
\begin{aligned}
& \sup _{x \in K-k}\left(1+x^{2}\right)^{N}\left|\left(\prod_{n=0}^{k-1} w(x+n)\right)^{(l)}\right| \\
& <C \varepsilon+M \sum_{i=1}^{l}\left(\begin{array}{l}
l \\
i
\end{array}\right) \sup _{x \in K-k}\left(1+x^{2}\right)^{N}\left|\left(\prod_{n=0}^{k-1} w(x+n)\right)^{(l-i)}\right| .
\end{aligned}
$$

This easily gives (using an induction argument over $l$ ) that there exists a positive constant $M$ such that for $0 \leq l \leq N$

$$
\sup _{x \in K-k}\left(1+x^{2}\right)^{N}\left|\left(\prod_{n=0}^{k-1} w(x+n)\right)^{(l)}\right|<M C \varepsilon
$$

Choosing the constant $C$ sufficiently small we get that the inequality in (1) is true for $0 \leq l \leq N$.

In order to prove the inequalities in (2) one needs to observe that if $g \in U$ is such that $f=T_{w}^{k}(g) \in U$, then for $x \in \mathbb{R}$

$$
g(x)=\frac{f(x-k)}{\prod_{n=1}^{k} w(x-n)} .
$$

Since $g \in U$, we get that for every $0 \leq l \leq N$

$$
\sup _{x \in K+k}\left(1+x^{2}\right)^{N}\left|g^{(l)}(x)\right| \leq \sup _{x \in \mathbb{R} \backslash[-D-1, D+1]}\left(1+x^{2}\right)^{N}\left|g^{(l)}(x)\right|<C \varepsilon
$$

and proceeding as earlier we obtain that the required inequalities hold.

(iii) $\Rightarrow$ (i) To show that $T_{w}$ is a weakly mixing operator let us take nonempty and open subsets $U_{1}, U_{2}, V_{1}, V_{2}$ of $\mathcal{S}(\mathbb{R})$. We need to show that there is $n \in \mathbb{N}$ such that

$$
T_{w}^{n}\left(U_{1}\right) \cap V_{1} \neq \varnothing \text { and } T_{w}^{n}\left(U_{2}\right) \cap V_{2} \neq \emptyset
$$

Since compactly supported smooth functions are dense in $\mathcal{S}(\mathbb{R})$, we can find compactly supported smooth functions $f_{1}, f_{2}, g_{1}, g_{2}$ and constants $\varepsilon>0, N \in \mathbb{N}$ such that

$$
\left\{h \in \mathcal{S}(\mathbb{R}):\left\|f_{i}-h\right\|_{N}<\varepsilon\right\} \subset U_{i} \text { and }\left\{h \in \mathcal{S}(\mathbb{R}):\left\|g_{i}-h\right\|_{N}<\varepsilon\right\} \subset V_{i}
$$

for $i=1,2$. Let us assume that the supports of the functions $f_{1}, f_{2}, g_{1}, g_{2}$ are contained in a compact set $K$ and let $\left(n_{k}\right)_{k \in \mathbb{N}}$ be an increasing sequence of natural numbers which existence is assumed in condition $(b)$. For $i=1,2$ we define the function $h_{i}$ via the formula 


$$
h_{i}(x)= \begin{cases}f_{i}(x), & x \in K, \\ \frac{g_{i}\left(x-n_{k}\right)}{\prod_{n=1}^{n_{k}} w(x-n)}, & x \in K+n_{k}, \\ 0, & \text { otherwise. }\end{cases}
$$

If $k$ is large enough, then the functions $h_{i}$ are well defined, compactly supported and smooth. For $i=1,2$, from the assumptions, using the product rule and the fact that the derivatives of $g_{i}$ are bounded we obtain that

$$
\left\|h_{i}-f_{i}\right\|_{N}=\max _{0 \leq j \leq N} \sup _{x \in K+n_{k}}\left(1+x^{2}\right)^{N}\left|\left(\frac{g_{i}\left(x-n_{k}\right)}{\prod_{n=1}^{n_{k}} w(x-n)}\right)^{(j)}\right| \stackrel{k \rightarrow \infty}{\longrightarrow} 0 .
$$

The same arguments show that for $i=1,2$

$$
\left\|T_{w}^{n_{k}} h_{i}-g_{i}\right\|_{N}=\max _{0 \leq j \leq N} \sup _{x \in K-n_{k}}\left(1+x^{2}\right)^{N}\left|\left(\prod_{n=0}^{n_{k}-1} w(x+n) f_{i}(x)\right)^{(j)}\right| \stackrel{k \rightarrow \infty}{\longrightarrow} 0 .
$$

Therefore, if $k$ is large enough, then for $i=1,2$ we have that $h_{i} \in U_{i}$ and $T_{w}^{n_{k}}\left(h_{i}\right) \in V_{i}$. This completes the proof.

The next theorem characterizes the class of mixing weighted translation operators on $\mathcal{S}(\mathbb{R})$. In Example 3 we will show that this class is strictly smaller than the class of hypercyclic operators (note that for weighted composition operators acting on the space of smooth functions on the real line those classes are equal, see [11]).

Theorem 2 Let $w \in \mathcal{O}_{M}(\mathbb{R})$. The following conditions are equivalent:

(i) The operator $T_{w}: \mathcal{S}(\mathbb{R}) \rightarrow \mathcal{S}(\mathbb{R}), f(\cdot) \mapsto w(\cdot) f(\cdot+1)$ is mixing.

(ii) The following conditions are satisfied:

(a) For every $x \in \mathbb{R}$ we have $w(x) \neq 0$

(b) For every compact set $K \subset \mathbb{R}$ and for every $j, l \geq 0$

$$
\sup _{x \in K-k}\left(1+x^{2}\right)^{j}\left|\left(\prod_{n=0}^{k-1} w(x+n)\right)^{(l)}\right| \stackrel{k \rightarrow \infty}{\longrightarrow} 0
$$

and

$$
\sup _{x \in K+k}\left(1+x^{2}\right)^{j}\left|\left(\frac{1}{\prod_{n=1}^{n_{k}} w(x-n)}\right)^{(l)}\right| \stackrel{k \rightarrow \infty}{\longrightarrow} 0 .
$$

The proof of this theorem is similar to the proof of Theorem 1. We include the proof for the convenience of the reader. 
Proof $(i) \Rightarrow$ (ii) Since every mixing operator is hypercyclic, we obtain from Theorem 1 that $w(x) \neq 0$ for every $x \in \mathbb{R}$. In order to show that the condition $(b)$ is satisfied let us fix $\varepsilon>0$ and $N \in \mathbb{N}$. We consider now the nonempty and open set $U$ from the proof of Theorem 1. Since $T_{w}$ is mixing, $T_{w}^{k}(U) \cap U \neq \emptyset$ for every $k$ large enough. Repeating the arguments from the previous proof we obtain that for $0 \leq l \leq N$ and $k$ large enough

$$
\sup _{x \in K-k}\left(1+x^{2}\right)^{N}\left|\left(\prod_{n=0}^{k-1} w(x+n)\right)^{(l)}\right|<\varepsilon
$$

and

$$
\sup _{x \in K+k}\left(1+x^{2}\right)^{N}\left|\left(\frac{1}{\prod_{n=1}^{k} w(x-n)}\right)^{(l)}\right|<\varepsilon
$$

This is enough.

(ii $) \Rightarrow(i)$ Let $U$ and $V$ be nonempty and open subsets of $\mathcal{S}(\mathbb{R})$. We need to show that $T_{w}^{k}(U) \cap V \neq \varnothing$ for every $k$ large enough. Since compactly supported smooth functions are dense in $\mathcal{S}(\mathbb{R})$, we can find compactly supported smooth functions $f, g$, constants $\varepsilon>0, N \in \mathbb{N}$ such that

$$
\left\{h \in \mathcal{S}(\mathbb{R}):\|f-h\|_{N}<\varepsilon\right\} \subset U \text { and }\left\{h \in \mathcal{S}(\mathbb{R}):\|g-h\|_{N}<\varepsilon\right\} \subset V .
$$

Let us assume that the supports of the functions $f, g$ are contained in a compact set $K$ and let us define the function $h$ via the formula

$$
h(x)= \begin{cases}f(x), & x \in K, \\ \frac{g(x-k)}{\prod_{n=1}^{n_{k}} w(x-n)}, & x \in K+k, \\ 0, & \text { otherwise. }\end{cases}
$$

If $k$ is large enough, then the function $h$ are well defined, compactly supported and smooth. Using the product rule and the fact that the derivatives of $g$ are bounded we obtain that

$$
\|h-f\|_{N}=\max _{0 \leq j \leq N} \sup _{x \in K+k}\left(1+x^{2}\right)^{N}\left|\left(\frac{g(x-k)}{\prod_{n=1}^{k} w(x-n)}\right)^{(j)}\right| \stackrel{k \rightarrow \infty}{\longrightarrow} 0 .
$$

The same arguments show that

$$
\left\|T_{w}^{k} h-g\right\|_{N}=\max _{0 \leq j \leq N} \sup _{x \in K-k}\left(1+x^{2}\right)^{N}\left|\left(\prod_{n=0}^{k-1} w(x+n) f(x)\right)^{(j)}\right| \stackrel{k \rightarrow \infty}{\longrightarrow} 0 .
$$


Therefore, if $k$ is large enough, then $h \in U$ and $T_{w}^{k}(h) \in V$.

The following result gives a description of chaotic weighted translation operators on $\mathcal{S}(\mathbb{R})$. Surprisingly, for those operators, the existence of a dense set of periodic points already implies that they are chaotic (a similar situation holds for weighted bilateral shifts, see [12]).

Theorem 3 Let $w \in \mathcal{O}_{M}(\mathbb{R})$. The following conditions are equivalent:

(i) The operator $T_{w}: \mathcal{S}(\mathbb{R}) \rightarrow \mathcal{S}(\mathbb{R}), f(\cdot) \mapsto w(\cdot) f(\cdot+1)$ is chaotic.

(ii) The operator $T_{w}: \mathcal{S}(\mathbb{R}) \rightarrow \mathcal{S}(\mathbb{R}), f(\cdot) \mapsto w(\cdot) f(\cdot+1)$ has a dense set of periodic points.

(iii) The following conditions are satisfied:

(a) For every $x \in \mathbb{R}$ we have $w(x) \neq 0$.

(b) For every compact set $K \subset \mathbb{R}$ there exists $d \in \mathbb{N}$ such that for every $j, l \geq 0$

$$
\sup _{x \in K-k d}\left(1+x^{2}\right)^{j}\left|\left(\prod_{n=0}^{k d-1} w(x+n)\right)^{(l)}\right| \stackrel{k \rightarrow \infty}{\longrightarrow} 0
$$

and

$$
\sup _{x \in K+k d}\left(1+x^{2}\right)^{j}\left|\left(\frac{1}{\prod_{n=1}^{k d} w(x-n)}\right)^{(l)}\right| \stackrel{k \rightarrow \infty}{\longrightarrow} 0 .
$$

Proof $(i) \Rightarrow$ (ii) This implication is obvious.

(ii) $\Rightarrow$ (iii) First we show that the condition $(a)$ holds. To do this let us assume that there exists $x_{0} \in \mathbb{R}$ such that $w\left(x_{0}\right)=0$. This implies that every periodic point of $T_{w}$ vanishes at $x_{0}$ which clearly implies that ( $i i$ ) cannot hold.

In order to prove that $(b)$ is satisfied let us take an arbitrary compact set $K \subset \mathbb{R}$. Condition ( $i$ i ) implies that there is $p \in \mathcal{S}(\mathbb{R})$ which is a periodic point for $T_{w}$ and satisfies the condition

$$
|p(x)|>1 \text { for } x \in K \text {. }
$$

We can find $d \in \mathbb{N}$ large enough to ensure that

$$
T_{w}^{d} p=p \text { and } K+n_{1} d \cap K+n_{2} d=\emptyset \text { for all integers } n_{1} \neq n_{2}
$$

Now, since $T_{w}^{k d} p=p$ for every $k \in \mathbb{N}$, it is easy to check that the function $p$ must satisfy the following equations

$$
p(x)= \begin{cases}\frac{p(x-k d)}{\prod_{n=1}^{k d} w(x-n)}, & x \in K+k d, k \geq 1, \\ \left(\prod_{n=0}^{k d-1} w(x+n)\right) \cdot p(x+k d), & x \in K-k d, k \geq 1 .\end{cases}
$$


Using induction with respect to $l$ we will show that for every $l \geq 0$ the following holds: for every $j \geq 0$

$$
\sup _{x \in K-k d}\left(1+x^{2}\right)^{j}\left|\left(\prod_{n=0}^{k d-1} w(x+n)\right)^{(l)}\right| \stackrel{k \rightarrow \infty}{\longrightarrow} 0
$$

and

$$
\sup _{x \in K+k d}\left(1+x^{2}\right)^{j}\left|\left(\frac{1}{\prod_{n=1}^{k d} w(x-n)}\right)^{(l)}\right| \stackrel{k \rightarrow \infty}{\longrightarrow} 0 .
$$

Step 1 Let $l=0$. Since $p \in \mathcal{S}(\mathbb{R})$, for every $j \geq 0$

$$
\sup _{x \in K-k d}\left(1+x^{2}\right)^{j}|p(x)| \stackrel{k \rightarrow \infty}{\longrightarrow} 0
$$

and

$$
\sup _{x \in K+k d}\left(1+x^{2}\right)^{j}|p(x)| \stackrel{k \rightarrow \infty}{\longrightarrow} 0 .
$$

Together with (7) this proves (9) and (10) for $l=0$ since

$$
\begin{aligned}
& \sup _{x \in K-k d}\left(1+x^{2}\right)^{j}\left|\prod_{n=0}^{k d-1} w(x+n)\right| \\
& <\sup _{x \in K-k d}\left(1+x^{2}\right)^{j}\left|\left(\prod_{n=0}^{k d-1} w(x+n)\right) p(x+k d)\right| \\
& =\sup _{x \in K-k d}\left(1+x^{2}\right)^{j}|p(x)|
\end{aligned}
$$

and

$$
\begin{aligned}
& \sup _{x \in K+k d}\left(1+x^{2}\right)^{j}\left|\frac{1}{\prod_{n=1}^{k d} w(x-n)}\right| \\
& <\sup _{x \in K+k d}\left(1+x^{2}\right)^{j}\left|\frac{p(x-k d)}{\prod_{n=1}^{k d} w(x-n)}\right| \\
& =\sup _{x \in K+k d}\left(1+x^{2}\right)^{j}|p(x)| .
\end{aligned}
$$

Step 2 Assume that the inequalities (9) and (10) are true for $0,1, \ldots, l-1$. We will show that they are true for $l$. 
Since $p \in \mathcal{S}(\mathbb{R})$, for every $j \geq 0$

$$
\sup _{x \in K-k d}\left(1+x^{2}\right)^{j}\left|p^{(l)}(x)\right| \stackrel{k \rightarrow \infty}{\longrightarrow} 0
$$

and

$$
\sup _{x \in K+k d}\left(1+x^{2}\right)^{j}\left|p^{(l)}(x)\right| \stackrel{k \rightarrow \infty}{\longrightarrow} 0 .
$$

Using (8) we get that

$$
\sup _{x \in K-k d}\left(1+x^{2}\right)^{j}\left|\left(\left(\prod_{n=0}^{k d-1} w(x+n)\right) p(x+k d)\right)^{(l)}\right| \stackrel{k \rightarrow \infty}{\longrightarrow} 0
$$

and

$$
\sup _{x \in K+k d}\left(1+x^{2}\right)^{j}\left|\left(\frac{p(x-k d)}{\prod_{k=1}^{n d} w(x-n)}\right)^{(l)}\right| \stackrel{k \rightarrow \infty}{\longrightarrow} 0 .
$$

By the product rule, the inductive hypothesis and the fact that $p$ and all its derivatives are bounded we get

$$
\sup _{x \in K-k d}\left(1+x^{2}\right)^{j}\left|\left(\prod_{n=0}^{k d-1} w(x+n)\right)^{(l)} p(x+k d)\right| \stackrel{k \rightarrow \infty}{\longrightarrow} 0
$$

and

$$
\sup _{x \in K+k d}\left(1+x^{2}\right)^{j}\left|\left(\frac{1}{\prod_{n=1}^{k d} w(x-n)}\right)^{(l)} p(x-k d)\right| \stackrel{k \rightarrow \infty}{\longrightarrow} 0 .
$$

From (7) it follows that $|p(x+k d)|>1$ for $x \in K-k d$ and $|p(x-k d)|>1$ for $x \in K+k d$. Together with (11) and (12) this proves the inductive hypothesis.

(iii) $\Rightarrow$ (i) By Theorem 1 the operator $T_{w}$ is hypercyclic and therefore we only need to show that $T_{w}$ has a dense set of periodic points. To do this we will prove that for every compactly supported smooth function $f$ there is a sequence $\left(p_{s}\right)_{s \in \mathbb{N}}$ of periodic points of $T_{w}$ which is convergent to $f$ in $\mathcal{S}(\mathbb{R})$.

Let $f \in \mathcal{S}(\mathbb{R})$ with supp $f \subset K=[-D, D]$. Let $d$ be a natural number for which conditions in (iii) are satisfied and such that $K \pm d \cap K=\emptyset$. For every $s \in \mathbb{N}$ we define a function $p_{s}: \mathbb{R} \rightarrow \mathbb{C}$ by the formula 


$$
p_{s}(x)= \begin{cases}f(x), & x \in K \\ \frac{f(x-n s d)}{\prod_{k=1}^{n s d} w(x-k)}, & x \in K+n s d, n \geq 1 \\ \left(\prod_{k=0}^{-n s d-1} w(x+k)\right) f(x-n s d), & x \in K+n s d, n \leq-1 \\ 0, & \text { otherwise. }\end{cases}
$$

The functions $p_{s}$ are smooth and will show that they are in $\mathcal{S}(\mathbb{R})$. In order to do this, let us fix $N \in \mathbb{N}$. From the definition of the functions $p_{s}$ it follows that

$$
\begin{aligned}
\left\|p_{s}\right\|_{N} & =\max _{0 \leq i \leq N} \sup _{x \in \mathbb{R}}\left(1+x^{2}\right)^{N}\left|p_{s}^{(i)}(x)\right| \\
& =\max _{0 \leq i \leq N} \sup _{n \in \mathbb{Z}} \sup _{x \in K+n s d}\left(1+x^{2}\right)^{N}\left|p_{s}^{(i)}(x)\right| .
\end{aligned}
$$

Since the function $f$ is compactly supported, there exists a constant $C$ such that

$$
\max _{0 \leq i \leq N} \sup _{x \in \mathbb{R}}\left|f^{(i)}(x)\right|<C
$$

Using this we obtain that

$$
\max _{0 \leq i \leq N} \sup _{x \in K}\left|p_{s}^{(i)}(x)\right|=\max _{0 \leq i \leq N} \sup _{x \in K}\left|f^{(i)}(x)\right|<C .
$$

Now using the assumptions, the inequality in (13) and the product rule we get that

$$
\begin{aligned}
& \max _{0 \leq l \leq N} \sup _{n \geq 1} \sup _{x \in K+n s d}\left(1+x^{2}\right)^{N}\left|p^{(l)}(x)\right| \\
& \quad=\max _{0 \leq l \leq N} \sup _{n \geq 1} \sup _{x \in K+n s d}\left(1+x^{2}\right)^{N}\left|\left(\frac{f(x-n s d)}{\prod_{k=1}^{n s d} w(x-k)}\right)^{(l)}\right| \\
& \quad<C \max _{0 \leq l \leq N} \sup _{n \geq 1} \sup _{x \in K+n s d} \sum_{j=0}^{l}\left(\begin{array}{l}
l \\
j
\end{array}\right)\left(1+x^{2}\right)^{N}\left|\left(\frac{1}{\prod_{k=1}^{n s d} w(x-k)}\right)^{(j)}\right|<\infty
\end{aligned}
$$

and

$$
\begin{aligned}
& \max _{0 \leq l \leq N} \sup _{n \leq-1} \sup _{x \in K+n s d}\left(1+x^{2}\right)^{N}\left|p_{s}^{(l)}(x)\right| \\
& \quad=\max _{0 \leq l \leq N} \sup _{n \leq-1} \sup _{x \in K+n s d}\left(1+x^{2}\right)^{N}\left|\left(\left(\prod_{k=0}^{-n s d-1} w(x+k)\right) f(x-n s d)\right)^{(l)}\right| \\
& \quad<C \max _{0 \leq l \leq N} \sup _{n \leq-1} \sup _{x \in K+n s d} \sum_{j=0}^{l}\left(\begin{array}{l}
l \\
j
\end{array}\right)\left(1+x^{2}\right)^{N}\left|\left(\prod_{k=0}^{-n s d-1} w(x+k)\right)^{(j)}\right|<\infty .
\end{aligned}
$$


This gives that $\left\|p_{s}\right\|_{N}<\infty$. Now, it is easy to see that for every $s \in \mathbb{N}$

$$
T_{w}^{s d} p_{s}=p_{s}
$$

and that the sequence $\left(p_{S}\right)_{s \in \mathbb{N}}$ converges to $f$ in $\mathcal{S}(\mathbb{R})$.

From Theorems 2 and 3 we immediately obtain the following corollary.

Corollary 1 Let $w \in \mathcal{O}_{M}(\mathbb{R})$. If $T_{w}: \mathcal{S}(\mathbb{R}) \rightarrow \mathcal{S}(\mathbb{R}), f(\cdot) \mapsto w(\cdot) f(\cdot+1)$ is mixing then it is chaotic.

We will show now that even more is true.

Theorem 4 Let $w \in \mathcal{O}_{M}(\mathbb{R})$. The following conditions are equivalent:

(i) The operator $T_{w}: \mathcal{S}(\mathbb{R}) \rightarrow \mathcal{S}(\mathbb{R}), f(\cdot) \mapsto w(\cdot) f(\cdot+1)$ is chaotic.

(ii) The operator $T_{w}: \mathcal{S}(\mathbb{R}) \rightarrow \mathcal{S}(\mathbb{R}), f(\cdot) \mapsto w(\cdot) f(\cdot+1)$ is mixing.

Proof By Corollary 1 it is enough to show that if $T_{w}$ is chaotic, then it is mixing. So let us assume that $T_{w}$ is chaotic. To prove that $T_{w}$ is mixing let us fix a compact set $K$. By Theorem 3 we know that $w(x) \neq 0$ for every $x \in \mathbb{R}$ and that there exists $d \in \mathbb{N}$ such that for every $j, l \geq 0$

$$
\sup _{x \in K-k d}\left(1+x^{2}\right)^{j}\left|\left(\prod_{n=0}^{k d-1} w(x+n)\right)^{(l)}\right| \stackrel{k \rightarrow \infty}{\longrightarrow} 0
$$

and

$$
\sup _{x \in K+k d}\left(1+x^{2}\right)^{j}\left|\left(\frac{1}{\prod_{n=1}^{k d} w(x-n)}\right)^{(l)}\right| \stackrel{k \rightarrow \infty}{\longrightarrow} 0 .
$$

Claim 1 For every $1 \leq s \leq d-1$ and every $j, l \geq 0$

$$
\sup _{x \in K-k d-s}\left(1+x^{2}\right)^{j}\left|\left(\prod_{n=0}^{k d+s-1} w(x+n)\right)^{(l)}\right| \stackrel{k \rightarrow \infty}{\longrightarrow} 0 .
$$

Proof of the claim. Let us fix $1 \leq s \leq d-1$ and $j, l \geq 0$. By the product rule

$$
\left(\prod_{n=0}^{k d+s-1} w(x+n)\right)^{(l)}=\sum_{i=0}^{l}\left(\begin{array}{l}
l \\
i
\end{array}\right)\left(\prod_{n=0}^{s-1} w(x+n)\right)^{(i)}\left(\prod_{n=s}^{k d+s-1} w(x+n)\right)^{(l-i)}
$$

Since $w \in \mathcal{O}_{M}(\mathbb{R})$, there exists $m \in \mathbb{N}$ such that for every $0 \leq j \leq l$ and every $x \in \mathbb{R}$ we have

$$
\left|w^{(j)}(x)\right|<m\left(1+x^{2}\right)^{m} .
$$


Therefore

$$
\left|\left(\prod_{n=0}^{k d+s-1} w(x+n)\right)^{(l)}\right| \leq C \prod_{n=0}^{s-1}\left(1+(x+n)^{2}\right)^{m} \sum_{i=0}^{l}\left|\left(\prod_{n=s}^{k d+s-1} w(x+n)\right)^{(l-i)}\right|
$$

where $C$ is a constant which does not depend on $k$. This gives that

$$
\begin{aligned}
& \sup _{x \in K-k d-s}\left(1+x^{2}\right)^{j}\left|\left(\prod_{n=0}^{k d+s-1} w(x+n)\right)^{(l)}\right| \\
& \leq C \sup _{x \in K-k d-s}\left(1+x^{2}\right)^{j} \prod_{n=0}^{s-1}\left(1+(x+n)^{2}\right)^{m} \sum_{i=0}^{l}\left|\left(\prod_{n=s}^{k d+s-1} w(x+n)\right)^{(l-i)}\right| \\
& =C \sup _{x \in K-k d}\left(1+(x-s)^{2}\right)^{j} \\
& \quad \times \prod_{n=0}^{s-1}\left(1+(x-s+n)^{2}\right)^{m} \sum_{i=0}^{l}\left|\left(\prod_{n=0}^{k d-1} w(x+n)\right)^{(l-i)}\right|
\end{aligned}
$$

Now, if $k$ is large enough and $x \in K-k d$, then

$$
\left(1+(x-s)^{2}\right)^{j} \prod_{n=0}^{s-1}\left(1+(x-s+n)^{2}\right)^{m} \leq\left(1+x^{2}\right)^{j+m s+1} .
$$

This proves (16) since by the above computations and by (14)

$$
\begin{aligned}
& \sup _{x \in K-k d-s}\left(1+x^{2}\right)^{j}\left|\left(\prod_{n=0}^{k d+s-1} w(x+n)\right)^{(l)}\right| \\
& \leq C \sup _{x \in K-k d}\left(1+x^{2}\right)^{j+m s+1} \sum_{i=0}^{l}\left|\left(\prod_{n=0}^{k d-1} w(x+n)\right)^{(l-i)}\right| \stackrel{k \rightarrow \infty}{\longrightarrow} 0 .
\end{aligned}
$$

Claim 2 For every $1 \leq s \leq d-1$

$$
\sup _{x \in K+k d-s}\left(1+x^{2}\right)^{j}\left|\left(\frac{1}{\prod_{n=1}^{k d-s} w(x-n)}\right)^{(l)}\right| \stackrel{k \rightarrow \infty}{\longrightarrow} 0 .
$$


Proof of the claim: The proof of this claim is very similar to the proof of Claim 1. One needs only to observe that

$$
\frac{1}{\prod_{n=1}^{k d-s} w(x-n)}=\frac{1}{\prod_{n=1}^{k d} w(x-n)} \cdot \prod_{n=k d-s+1}^{k d} w(x-n) .
$$

Claim 1 and Claim 2 easily give us that

$$
\sup _{x \in K-k}\left(1+x^{2}\right)^{j}\left|\left(\prod_{n=0}^{k-1} w(x+n)\right)^{(l)}\right| \stackrel{k \rightarrow \infty}{\longrightarrow} 0
$$

and

$$
\sup _{x \in K+k}\left(1+x^{2}\right)^{j}\left|\left(\frac{1}{\prod_{n=1}^{k d} w(x-n)}\right)^{(l)}\right| \stackrel{k \rightarrow \infty}{\longrightarrow} 0 .
$$

By Theorem 2 this implies that $T_{w}$ is mixing.

\section{Examples}

The aim of this section is to illustrate our results and to show which of the considered classes of weighted translation operators on $\mathcal{S}(\mathbb{R})$ are different. We start with the following proposition which gives a wide class of examples of mixing and chaotic weighted translation operators.

Proposition 1 Let $w \in \mathcal{O}_{M}(\mathbb{R})$ be such that $w(x) \neq 0$ for every $x \in \mathbb{R}$.

(i) If there exists $0<c<1$ and $n_{0} \in \mathbb{N}$ such that $|w(x)|<c$ for $x \leq-n_{0}$, then for every compact set $K \subset \mathbb{R}$

$$
\sup _{x \in K-k}\left(1+x^{2}\right)^{j}\left|\left(\prod_{n=0}^{k-1} w(x+n)\right)^{(l)}\right| \stackrel{k \rightarrow \infty}{\longrightarrow} 0 \text { for every } j, l \geq 0 .
$$

(ii) If there exists $C>1$ and $n_{0} \in \mathbb{N}$ such that $|w(x)|>C$ for $x \geq n_{0}$, then for every compact set $K \subset \mathbb{R}$

$$
\sup _{x \in K+k}\left(1+x^{2}\right)^{j}\left|\left(\frac{1}{\prod_{n=1}^{k} w(x-n)}\right)^{(l)}\right| \stackrel{k \rightarrow \infty}{\longrightarrow} 0 \text { for every } j, l \geq 0 .
$$

(iii) If there exists $0<\delta<1$ and $n_{0} \in \mathbb{N}$ such that $|w(x)|<\delta$ for $x \leq-n_{0}$ and $|w(x)|>\frac{1}{\delta}$ for $x \geq n_{0}$, then the operator $T_{w}: \mathcal{S}(\mathbb{R}) \rightarrow \mathcal{S}(\mathbb{R}), f(\cdot) \mapsto$ $w(\cdot) f(\cdot+1)$ is mixing and chaotic. 
Proof $(i)$ Without loss of generality we can take $K=[-D, D]$, where $D \in \mathbb{N}$. In order to show that (17) holds let us first observe that if $k \geq D+n_{0}+1$, then

$$
\begin{aligned}
& \left(\prod_{n=0}^{k-1} w(x+n)\right)^{(l)} \\
& =\sum_{i=0}^{l}\left(\begin{array}{l}
l \\
i
\end{array}\right)\left(\prod_{n=0}^{k-D-n_{0}} w(x+n)\right)^{(l-i)}\left(\prod_{n=k-D-n_{0}+1}^{k-1} w(x+n)\right)^{(i)} .
\end{aligned}
$$

Since $w$ is smooth, for every every $i \geq 0$ there exists $C_{i}>0$ such that for every $k \in \mathbb{N}$

$$
\begin{aligned}
& \sup _{x \in[-D, D]-k}\left|\left(\prod_{n=k-D-n_{0}+1}^{k-1} w(x+n)\right)^{(i)}\right| \\
& =\sup _{x \in[-D, D]}\left|\left(\prod_{n=-D-n_{0}+1}^{-1} w(x+n)\right)^{(i)}\right| \leq C_{i} .
\end{aligned}
$$

Therefore, to prove that (17) holds it is enough to show that

$$
\sup _{x \in[-D, D]-k}\left(1+x^{2}\right)^{j}\left|\left(\prod_{n=0}^{k-D-n_{0}} w(x+n)\right)^{(l)}\right| \stackrel{k \rightarrow \infty}{\longrightarrow} 0 \text { for every } j, l \geq 0
$$

By the product rule we get that

$$
\begin{aligned}
& \left(\prod_{n=0}^{k-D-n_{0}} w(x+n)\right)^{(l)} \\
& =\sum_{\substack{i_{0}, \ldots, i_{k-D-n_{0}} \geq 0 \\
i_{0}+\cdots+i_{k-D-n_{0}}=l}} \frac{l !}{i_{0} ! \ldots i_{k-D-n_{0}} !} \prod_{n=0}^{k-D-n_{0}} w^{\left(i_{n}\right)}(x+n) .
\end{aligned}
$$

Let $i_{0}, \ldots, i_{k-D-n_{0}} \geq 0$ be such that $i_{0}+\cdots+i_{k-D-n_{0}}=l$. If $x \in[-D, D]-k$, then for $n=0, \ldots, k-D-n_{0}$ we have that $-D-k \leq x+n \leq-n_{0}$. Since $w \in \mathcal{O}_{M}(\mathbb{R})$ there exists $m \in \mathbb{N}$ such that for every $0 \leq j \leq l$ and every $x \in \mathbb{R}$ we have $\left|w^{(j)}(x)\right|<m\left(1+x^{2}\right)^{m}$. Using the assumptions we get that for $x \in[-D, D]-k$ and $k$ large enough 


$$
\begin{aligned}
\left|\prod_{n=0}^{k-D-n_{0}} w^{\left(i_{n}\right)}(x+n)\right|=\left|\prod_{\substack{0 \leq n \leq k-D-n_{0} \\
i_{n}=0}} w(x+n)\right| \cdot\left|\prod_{\substack{0 \leq n \leq k-D-n_{0} \\
i_{n} \neq 0}} w^{\left(i_{n}\right)}(x+n)\right| \\
\leq c^{k-D-n_{0}+1-l} \cdot m^{l} \cdot\left(1+(-D-k)^{2}\right)^{m l} .
\end{aligned}
$$

Thus, from (20), for $x \in[-D, D]-k$ and $k$ large enough we get

$$
\begin{aligned}
& \left|\left(\prod_{n=0}^{k-D-n_{0}} w(x+n)\right)^{(l)}\right| \\
& \quad \leq\left(k-D-n_{0}+1\right)^{l} \cdot l ! \cdot c^{k-D-n_{0}+1-l} \cdot m^{l} \cdot\left(1+(-D-k)^{2}\right)^{m l} .
\end{aligned}
$$

Therefore

$$
\begin{aligned}
& \sup _{x \in[-D, D]-k}\left(1+x^{2}\right)^{j}\left|\left(\prod_{n=1}^{k-D-n_{0}} w(x+n)\right)^{(l)}\right| \\
& \leq\left(1+(-D-k)^{2}\right)^{j} \cdot\left(k-D-n_{0}+1\right)^{l} \cdot l ! \\
& \quad \times c^{k-D-n_{0}+1-l} \cdot m^{l} \cdot\left(1+(-D-k)^{2}\right)^{m l} \stackrel{k \rightarrow \infty}{\longrightarrow} 0 .
\end{aligned}
$$

This proves (19).

(ii) The proof of this part is similar to the proof of the first part of the proposition. The only difference is that one needs to use the formula for higher derivatives of the function $\frac{1}{w(x+n)}$.

(iii) The last assertion in the proposition follows immediately from the first two parts, Theorems 2 and 3.

Example 1 Let $w \in \mathcal{O}_{M}(\mathbb{R})$ be such that $w(x) \neq 0$ for $x \in \mathbb{R}$ and satisfies $\lim _{x \rightarrow-\infty}|w(x)|=0$ and $\lim _{x \rightarrow \infty}|w(x)|=\infty$. Then, by Proposition 1 , the operator $T_{w}: \mathcal{S}(\mathbb{R}) \rightarrow \mathcal{S}(\mathbb{R}), f(\cdot) \mapsto w(\cdot) f(\cdot+1)$ is mixing and chaotic.

Example 2 Let $w: \mathbb{R} \rightarrow \mathbb{R}$ be any smooth function such that $w(x)=\frac{1}{2}$ if $x \leq 0$, $w(x)=2$ if $x \geq 1$ and $w(x) \neq 0$ for $x \in[0,1]$. It is clear that such a function belongs to $\mathcal{O}_{M}(\mathbb{R})$. By Proposition 1 , the operator $T_{w}: \mathcal{S}(\mathbb{R}) \rightarrow \mathcal{S}(\mathbb{R}), f(\cdot) \mapsto w(\cdot) f(\cdot+1)$ is mixing and chaotic.

The following proposition is an useful tool to construct examples of weighted translation operators with various dynamical properties.

Proposition 2 Let $w \in \mathcal{O}_{M}(\mathbb{R})$ be such that $w(x) \neq 0$ for every $x \in \mathbb{R}$ and let $K=[-D, D]$, where $D \in \mathbb{N}$. 
(i) Assume that there exist $c>0$ and $n_{0} \in \mathbb{N}$ such that $|w(x)|>c$ for $x \leq-n_{0}$. Let $\left(n_{k}\right)_{k \in \mathbb{N}}$ be an increasing sequence of natural numbers such that for every $j \geq 0$

$$
\sup _{x \in K-n_{k}}\left(1+x^{2}\right)^{j}\left|\prod_{n=0}^{n_{k}-1} w(x+n)\right| \stackrel{k \rightarrow \infty}{\longrightarrow} 0
$$

Then for every $j, l \geq 0$

$$
\sup _{x \in K-n_{k}}\left(1+x^{2}\right)^{j}\left|\left(\prod_{n=0}^{n_{k}-1} w(x+n)\right)^{(l)}\right| \stackrel{k \rightarrow \infty}{\longrightarrow} 0 .
$$

(ii) Assume that there exist $C>0$ and $n_{0} \in \mathbb{N}$ such that $|w(x)| \leq C$ for $x \geq n_{0}$. Let $\left(n_{k}\right)_{k \in \mathbb{N}}$ be an increasing sequence of natural numbers such that for every $j \geq 0$

$$
\sup _{x \in K+n_{k}}\left(1+x^{2}\right)^{j}\left|\frac{1}{\prod_{n=1}^{n_{k}} w(x-n)}\right| \stackrel{k \rightarrow \infty}{\longrightarrow} 0 .
$$

Then for every $j, l \geq 0$

$$
\sup _{x \in K+n_{k}}\left(1+x^{2}\right)^{j}\left|\left(\frac{1}{\prod_{n=1}^{n_{k}} w(x-n)}\right)^{(l)}\right| \stackrel{k \rightarrow \infty}{\longrightarrow} 0 .
$$

Proof $(i)$ Proceeding exactly the same way as in the beginning of the proof of the first part of Proposition 1 we get that the assumptions imply that

$$
\sup _{x \in[-D, D]-n_{k}}\left(1+x^{2}\right)^{j}\left|\prod_{n=0}^{\mid n_{k}-D-n_{0}} w(x+n)\right| \stackrel{k \rightarrow \infty}{\longrightarrow} 0 \text { for every } j \geq 0 .
$$

Moreover, in the same way, we obtain that to prove the desired statement it is enough to show that

$$
\sup _{x \in[-D, D]-n_{k}}\left(1+x^{2}\right)^{j}\left|\left(\prod_{n=0}^{n_{k}-D-n_{0}} w(x+n)\right)^{(l)}\right| \stackrel{k \rightarrow \infty}{\longrightarrow} 0 \text { for every } j, l \geq 0 .
$$


By the product rule we get that

$$
\begin{aligned}
& \left(\prod_{n=0}^{n_{k}-D-n_{0}} w(x+n)\right)^{(l)} \\
& =\sum_{\substack{i_{0}, \ldots, i_{n_{k}-D-n_{0}} \geq 0 \\
i_{0}+\cdots+i_{n_{k}}-D-n_{0}}} \frac{l !}{i_{0} ! \ldots i_{n_{k}-D-n_{0}} !} \prod_{n=0}^{n_{k}-D-n_{0}} w^{\left(i_{n}\right)}(x+n) \\
& =\sum_{\substack{i_{0}, \ldots, i_{n_{k}-D-n_{0}} \geq 0 \\
i_{0}+\cdots+i_{n_{k}}-D-n_{0}}} \frac{l !}{i_{0} ! \ldots i_{n_{k}-D-n_{0}} !}\left(\prod_{n=0}^{n_{k}-D-n_{0}} w(x+n)\right) \\
& \quad \times\left(\prod_{\substack{0 \leq n \leq n_{k}-D-n_{0} \\
i_{n} \neq 0}} \frac{w^{\left(i_{n}\right)}(x+n)}{w(x+n)}\right)
\end{aligned}
$$

Let $i_{0}, \ldots, i_{n_{k}-D-n_{0}} \geq 0$ be such that $i_{0}+\cdots+i_{n_{k}-D-n_{0}}=l$. If $x \in[-D, D]-n_{k}$, then for $n=0, \ldots, n_{k}-D-n_{0}$ we have that $-D-n_{k} \leq x+n \leq-n_{0}$. Since $w \in \mathcal{O}_{M}(\mathbb{R})$ there exists $m \in \mathbb{N}$ such that for every $0 \leq j \leq l$ and every $x \in \mathbb{R}$ we have $\left|w^{(j)}(x)\right|<m\left(1+x^{2}\right)^{m}$. Using the assumptions we get that for $x \in[-D, D]-n_{k}$ and $k$ large enough

$$
\left|\prod_{\substack{0 \leq n \leq n_{k}-D-n_{0} \\ i_{n} \neq 0}} \frac{w^{\left(i_{n}\right)}(x+n)}{w(x+n)}\right| \leq \frac{m^{l}}{c^{l}}\left(1+\left(-D-n_{k}\right)^{2}\right)^{m l} .
$$

Therefore

$$
\begin{aligned}
& \left|\left(\prod_{n=0}^{n_{k}-D-n_{0}} w(x+n)\right)^{(l)}\right| \\
& \leq\left|\prod_{n=0}^{n_{k}-D-n_{0}} w(x+n)\right| \cdot\left(n_{k}-D-n_{0}+1\right)^{l} \cdot l ! \cdot \frac{m^{l}}{c^{l}}\left(1+\left(-D-n_{k}\right)^{2}\right)^{m l} .
\end{aligned}
$$

For $x \in[-D, D]-n_{k}$ and $k$ large enough we have that

$$
\left(n_{k}-D-n_{0}+1\right)^{l} \cdot l ! \cdot \frac{m^{l}}{c^{l}}\left(1+\left(-D-n_{k}\right)^{2}\right)^{m l} \leq\left(1+x^{2}\right)^{m l+l+1} .
$$


Thus, from our calculations we get that

$$
\begin{aligned}
& \sup _{x \in[-D, D]-n_{k}}\left(1+x^{2}\right)^{j}\left|\left(\prod_{n=0}^{n_{k}-D-n_{0}} w(x+n)\right)^{(l)}\right| \\
& \leq \sup _{x \in[-D, D]-n_{k}}\left(1+x^{2}\right)^{j+m l+l+1}\left|\prod_{n=0}^{n_{k}-D-n_{0}} w(x+n)\right| .
\end{aligned}
$$

By our assumptions, the right hand side goes to zero as $k$ goes to infinity. This proves (22).

(ii) The proof of this part is similar to the proof of the first part of the theorem. The only difference is that one needs to use the formula for higher derivatives of the function $\frac{1}{w(x+n)}$.

In the following example we construct a weighted translation operator on $\mathcal{S}(\mathbb{R})$ which is hypercyclic and is not mixing (and thus not chaotic).

Example 3 Let $w \in \mathcal{O}_{M}(\mathbb{R})$ be a real valued function with the following properties:

(i) $w(x)=2$ for $x \geq-2$ and for $x \in\left[-2^{k+1},-2^{k}-2^{k-1}-1\right]$ for $k \in \mathbb{N}$;

(ii) $w(x)=1 / 2$ for $x \in\left[-2^{k}-2^{k-1},-2^{k}-1\right]$ for $k \in \mathbb{N}$

(iii) $w$ is decreasing on $\left[-2^{k}-2^{k-1}-1,-2^{k}-2^{k-1}\right]$ for $k \in \mathbb{N}$;

(iv) $w$ is increasing on $\left[-2^{k}-1,-2^{k}\right]$ for $k \in \mathbb{N}$;

(v) $w(x) w\left(x-2^{k-1}\right)=1$ for $x \in\left[-2^{k}-1,-2^{k}\right]$ and $k \in \mathbb{N}$.

It is clear that we can find such a function in $\mathcal{O}_{M}(\mathbb{R})$. From Proposition 1 we obtain that for every compact set $K \subset \mathbb{R}$

$$
\sup _{x \in K+k}\left(1+x^{2}\right)^{j}\left|\left(\frac{1}{\prod_{n=1}^{k} w(x-n)}\right)^{(l)}\right| \stackrel{k \rightarrow \infty}{\longrightarrow} 0 \quad \text { for every } j, l \geq 0 .
$$

Now, if $K=[-D, D]$, where $D \in \mathbb{N}$, then one can easily check that that for every $j \geq 0$

$$
\sup _{x \in[-D, D]-2^{k}-2^{k-1}+D}\left(1+x^{2}\right)^{j}\left|\prod_{n=0}^{2^{k}+2^{k-1}-D-1} w(x+n)\right| \stackrel{k \rightarrow \infty}{\longrightarrow} 0 .
$$

By Proposition 2 we get that

$$
\sup _{x \in[-D, D]-2^{k}-2^{k-1}+D}\left(1+x^{2}\right)^{j}\left|\left(\prod_{n=0}^{2^{k}+2^{k-1}-D-1} w(x+n)\right)^{(l)}\right| \stackrel{k \rightarrow \infty}{\longrightarrow} 0
$$


for every $j, l \geq 0$. By Theorem 1 , this gives that $T_{w}$ is hypercyclic. Let us now observe that for $K=\{-1\}$ we have that

$$
\sup _{x \in K-2^{k}} \prod_{n=0}^{2^{k}-1} w(x+n)=\prod_{n=0}^{2^{k}-1} w\left(-2^{k}-1+n\right)=1 .
$$

This shows that $T_{w}$ is not a mixing operator and thus also not chaotic.

Open Access This article is distributed under the terms of the Creative Commons Attribution 4.0 International License (http://creativecommons.org/licenses/by/4.0/), which permits unrestricted use, distribution, and reproduction in any medium, provided you give appropriate credit to the original author(s) and the source, provide a link to the Creative Commons license, and indicate if changes were made.

\section{References}

1. Bayart, F., Matheron, É.: Dynamics of Linear Operators, Volume 179 of Cambridge Tracts in Mathematics. Cambridge University Press, Cambridge (2009)

2. Birkhoff, G .D.: Démonstration d'un théorème élémentaire sur les fonctions entières. C. R. Acad. Sci. Paris 189, 473-475 (1929)

3. Bonet, J., Domański, P.: Hypercyclic composition operators on spaces of real analytic functions. Math. Proc. Camb. Philos. Soc. 153(3), 489-503 (2012)

4. Bourdon, P.S., Shapiro, J.H.: Hypercyclic operators that commute with the Bergman backward shift. Trans. Am. Math. Soc. 352(11), 5293-5316 (2000)

5. de la Rosa, M., Read, C.: A hypercyclic operator whose direct sum $T \oplus T$ is not hypercyclic. J. Oper. Theory 61(2), 369-380 (2009)

6. Galbis, A., Jordá, E.: Composition operators on the Schwartz space. Rev. Mat. Iberoam. 34(1), 397-412 (2018)

7. Grosse-Erdmann, K.-G.: Hypercyclic and chaotic weighted shifts. Stud. Math. 139(1), 47-68 (2000)

8. Grosse-Erdmann, K.-G., Mortini, R.: Universal functions for composition operators with nonautomorphic symbol. J. Anal. Math. 107, 355-376 (2009)

9. Grosse-Erdmann, K.-G., Peris Manguillot, A.: Linear Chaos. Universitext. Springer, London (2011)

10. Kalmes, T.: Hypercyclic, mixing, and chaotic $C_{0}$-semigroups induced by semiflows. Ergod. Theory Dyn. Syst. 27(5), 1599-1631 (2007)

11. Przestacki, A.: Dynamical properties of weighted composition operators on the space of smooth functions. J. Math. Anal. Appl. 445(1), 1097-1113 (2017)

12. Salas, H.N.: Hypercyclic weighted shifts. Trans. Am. Math. Soc. 347(3), 993-1004 (1995)

Publisher's Note Springer Nature remains neutral with regard to jurisdictional claims in published maps and institutional affiliations. 